\title{
Enurese: Impacto em Crianças e Adolescentes e a Tolerância dos Pais
}

\author{
Yasmin Spaolonzi Daibs \\ Rodrigo Fernando Pereira \\ Edwiges Ferreira de Mattos Silvares \\ Universidade de São Paulo \\ Deisy Ribas Emerich \\ Universidade Presbiteriana Mackenzie
}

\begin{abstract}
RESUMO
A literatura aponta que crianças e adolescentes com enurese podem ser impactados por esse problema e seus pais podem reagir de forma intolerante à condição dos filhos. O objetivo deste estudo foi verificar se havia relação entre a intolerância dos pais e o impacto percebido pelos filhos. Verificou-se também se os níveis de impacto e de tolerância materna são diferentes entre crianças e adolescentes. Participaram 126 crianças e adolescentes, e seus pais. As crianças responderam a uma Escala de Impacto, enquanto seus pais preencheram a Escala de Intolerância. Foi encontrada correlação positiva entre a intolerância da mãe e o impacto sentido pela criança. Verificou-se também que tanto o impacto como a intolerância apresentam correlação positiva com a idade. Estes resultados indicam que o tratamento da enurese, abrangendo em conjunto os clientes e seus pais, com esclarecimentos de ambos sobre as causas da enurese e a ausência de culpa das crianças que não conseguem obter o controle vesical pode minimizar o sofrimento de ambos, mas especialmente dos primeiros.
\end{abstract}

Palavras-chave: enurese; impacto psicossocial; intolerância; relações pais-filhos.

\section{ABSTRACT \\ Enuresis: Impact on Children and Adolescents and Parents' Tolerance}

Literature shows that enuresis can affect children and adolescents that suffer from this problem. It also can lead parents to an intolerant reaction toward their children condition. The objective of this study was to verify the relations between the impact perceived by the child and the parents' level of tolerance. Correlations between these two aspects and age were also investigated. One hundred twenty-six children and their parents participated in this study, filling the Impact Scale and the Tolerance Scale, respectively. A positive correlation between mothers' intolerance and children impact was found. There were also a positive correlation between both instruments scores and age. These results show that enuresis treatment should involve the whole family. It is important to clarify, both for parents and children, the causes of bedwetting and inform them that the child is not to be blamed by his or her lack of urinary control. This way, parents and their children's suffering may be reduced.

Keywords: enuresis; psychosocial impact; intolerance; parent-child relations.

Nos últimos anos, a discussão sobre os aspectos psicológicos associados à enurese tem sido foco de diversas publicações internacionais, mas poucas nacionais. Uma vez que a compreensão da etiologia da enurese atingiu um nível satisfatório (Nevéus, 2009) e os tratamentos disponíveis, tanto psicológicos como farmacológicos, têm obtido um bom nível de sucesso, os pesquisadores voltaram sua atenção para os problemas que a condição pode causar para a criança e para suas famílias, bem como os benefícios do tratamento para a vida familiar.
Porém, antes de entrarmos propriamente no tema desse artigo, é importante esclarecer o conceito de enurese. Existem dois critérios usados amplamente para identificar a enurese: um elaborado pela Associação Psiquiátrica Americana (APA) e outro pela Sociedade Internacional de Continência da Criança (ICCS). O Manual Diagnóstico e Estatístico de Transtornos Mentais - DSM-IV (American Psychiatric Association, 2002) estabelece quatro critérios para diagnóstico: (a) micção repetida, diurna ou noturna, na cama ou na 
roupa; (b) a micção deve ocorrer no mínimo duas vezes por semana por pelo menos três meses, ou então causar um sofrimento ou prejuízo significativo no funcionamento social, acadêmico (ocupacional) ou outras áreas importantes na vida do indivíduo; (c) idade cronológica de, no mínimo, cinco anos, ou para crianças com atrasos de desenvolvimento, idade mental de no mínimo cinco anos; (d) a incontinência urinária não se deve exclusivamente aos efeitos fisiológicos diretos de uma substância (por exemplo, diuréticos) ou a uma condição geral (por exemplo, diabete, espinha bífida, transtorno convulsivo).

Já a proposta de padronização apresentada pela International Child Continency Society - ICCS (Nevéus $\&$ cols., 2006) coloca que a enurese é definida como a micção normal em local ou hora inadequada. Em relação à idade, o critério é que a criança tenha mais de cinco anos. Contudo, diferentemente do DSM-IV, os autores estabelecem como critério para definição de enurese uma frequência de pelo menos uma vez ao mês. Além disso, apontam a necessidade de que a quantidade de urina evidente na cama da criança pela manhã seja grande, uma vez que pequenas quantidades podem indicar outros problemas que não a enurese.

Especialmente fruto dessa discrepância, os estudos sobre a prevalência da enurese tendem a apresentar resultados discordantes, já que não há uniformidade principalmente na definição da frequência de episódios estabelecida para se considerar que uma criança apresenta enurese. Essa falta de uniformidade leva autores a afirmações contraditórias ao longo de suas publicações. Por exemplo, Butler, em 1994, havia afirmado que de $13 \%$ a $19 \%$ dos meninos e entre $9 \%$ e $16 \%$ das meninas de cinco anos de idade molhavam suas camas, sendo que essa taxa diminuía durante a infância e a adolescência. Na mesma publicação, o autor propõe ainda que cerca de $2 \%$ a $3 \%$ dos adultos jovens ainda continuariam perdendo urina durante o sono. Sabidamente, porém, mesmo sem tratamento, cerca de $15 \%$ dos portadores de enurese obtém o controle vesical a cada ano, o que é chamado de remissão espontânea (Jensen \& Kristensen, 2001). Um estudo mais recente (Butler, Golding, Northstone \& ALSPAC Study Team, 2005) aponta que $15,5 \%$ das crianças de sete anos e meio de idade molham suas camas, mas a maior parte delas faz isso menos de uma vez por semana. Apenas 2,6\% das crianças dessa idade atingem o critério para enurese definido pelo DSM-IV.

O mesmo tipo de contradição pode também ser encontrado em publicações sobre enurese no Brasil.
Assim, um trabalho realizado por Schoen-Ferreira, Marteleto, Medeiros, Fisberg e Aznar-Farias (2007), utilizando um instrumento global de avaliação dirigido aos pais e que continha uma questão sobre enurese, o Child Behavior Checklist - CBCL (Achenbach \& Rescorla, 2001), mostrou que o relato da existência de episódios ocorreu para $11,3 \%$ das crianças em nível escolar, 6,6\% dos adolescentes e 1,8\% dos jovens. Mota, Victora e Hallal (2005), em um estudo brasileiro sobre prevalência da enurese na região de Pelotas, com amostra probabilística obtida diretamente em domicílios, identificaram uma prevalência de enurese em $20,1 \%$ dos meninos e $15,1 \%$ das meninas de até 9 anos de idade (o critério utilizado para definição de enurese foi o da ICCS). Daí a importância de os estudiosos deixarem claro em suas publicações quais os critérios utilizados para definição da enurese. As crianças objeto desse estudo foram consideradas portadoras de enurese segundo o critério do DSM-IV.

Quanto à etiologia, Butler e Holland (2000) apresentaram um modelo denominado "três sistemas" que indica os possíveis mecanismos fisiológicos por trás da enurese: (a) falta de liberação do hormônio responsável pela redução da produção de urina durante o sono, a vasopressina; (b) instabilidade da bexiga e (c) incapacidade de acordar ou contrair a musculatura pélvica em resposta às sensações de bexiga cheia. Um estudo realizado por pesquisadores belgas (Baeyens, Roeyers, Naert, Hoebeke \& Walle, 2007) evidencia que de fato as crianças com o transtorno têm um déficit maturacional no núcleo responsável pela inibição das contrações involuntárias da bexiga, impossibilitando a resposta que lhes permitiria continuar dormindo sem que a perda involuntária de urina ocorra.

Houts (1991) aponta os três principais tipos de tratamento medicamentoso para enurese: Imipramina (Tofranil), que age sobre a musculatura da bexiga, apresentando um bom resultado a curto prazo, mas que não se mantém ao longo do tempo; Oxibutinina (Ditropan), que também age na musculatura detrussora, mas apresenta um resultado inferior ao da Imipramina, e se mantém apenas a curto prazo; Desmopressina (DDAVP), que age nos túbulos distais do rim a fim de concentrar a urina e diminuir o volume produzido durante a noite, apresentando resultados similares aos outros medicamentos.

O uso do alarme de urina, um tratamento comportamental, em oposição às opções medicamentosas, é recomendado como uma alternativa, cuja efetividade situa-se em torno de $60 \%$ a $70 \%$ dos casos (Butler, 
2004), mesmo que a criança já tenha passado por outros tratamentos (Van Hoeck \& cols., 2007). Quando esse tipo de tratamento é realizado em conjunto com um procedimento de prevenção de recaída, a taxa de retorno dos sintomas é de 10\% (Houts, 2003), dando ao tratamento com alarme uma eficácia significativa também a longo prazo.

Embora se saiba que a prevalência da enurese é alta e que há relativa clareza sobre os aspectos etiológicos e de tratamento, ainda existem controvérsias na literatura sobre os aspectos psicológicos das crianças portadoras de enurese. Butler (1998) afirma que a enurese pode ser um problema devastador e incapacitante socialmente. $\mathrm{O}$ autor acrescenta ainda que, se por um lado há poucas evidências de que ela é causada por questões emocionais, o contrário parece verdadeiro. Butler (2001) também afirma que a enurese afeta a criança em diversas áreas, como: (a) comportamento social, marcado pela atitude evitativa em relação a diversas atividades; (b) estado emocional, demonstrado por relatos de infelicidade e sofrimento; (c) personalidade, ou seja, um sentimento de ser dessemelhante aos outros e d) comportamento, área em que essas crianças apresentariam mais problemas que outras.

Uma pesquisa nacional (Santos \& Silvares, 2006) apontou que as crianças portadoras de enurese avaliadas pelo CBCL obtêm escores de competências superiores e escores de problemas de comportamento inferiores aos das demais crianças encaminhadas para a clínica psicológica. As autoras defendem a tese de que a enurese é um problema primário, ou seja, dela derivam as outras dificuldades das crianças. Um estudo populacional mostrou que as crianças portadoras de enurese não apresentam menor autoestima quando comparadas com as que não molham a cama. No entanto, seus pais relataram um índice significativamente maior de distúrbios externalizantes, como comportamentos opositores e problemas de conduta (Joinson, Heron \& Butler, 2007).

Robinson, Butler, Holland e Doherty-Williams (2003) compararam 25 crianças com enurese (pelo menos três episódios por semana) com 25 crianças sem o problema, que formaram o grupo controle. Foram aplicadas três escalas, medindo a identidade pessoal, autoestima e a autoimagem positiva. Não houve diferença significativa entre o grupo de portadores de enurese e o controle em nenhuma delas. Uma das hipóteses para a dificuldade na identificação desses problemas é que talvez considerar o grupo de portado- res de enurese como um todo pode não ser a melhor abordagem, como mostram Collier, Butler, Redsell e Evans (2002). No estudo desses autores, notou-se que os mais afetados em relação à autoimagem eram meninos que tinham uma quantidade maior de episódios.

Aparentemente, o impacto da enurese percebido pelas próprias crianças como problema aumenta conforme a idade (Theunis, Van Hoecke, Paesbrugge, Hoebeke \& Walle, 2002). Outra evidência de que a enurese de fato traz problemas para seus portadores é que, após um tratamento bem sucedido, as crianças obtêm melhoras significativas nos escores de problemas de comportamento medidos pelo CBCL e de autoimagem (Longstaffe, Moffatt \& Whalen, 2000). No Brasil, Pereira, Del Prette e Silvares (2008), em estudo sobre a utilidade do índice de mudança confiável, no contexto de intervenção clínica com enurese dentro das instituições brasileiras, puderam demonstrar mudanças clínicas significativas nos comportamentos de clientes que concluíram o tratamento com o aparelho de alarme de urina. No entanto, a questão de esses indivíduos apresentarem ou não mais problemas do que os que não têm enurese ainda é controversa e inconclusiva.

Outra forma de contradição é aparente no trabalho de Van Hoecke, Hoebeke, Braet e Walle (2004) em que os pais de crianças portadoras de enurese classificaram como tendo mais problemas internalizantes do que os pais de crianças que não tinham o transtorno. No entanto, os questionários respondidos pelas próprias crianças não tiveram diferença entre os grupos de participantes com e sem enurese. Tais dados são confirmados por um recente estudo brasileiro de Rocha, Costa e Silvares (2008).

Estudos sobre o impacto causado pela enurese são justificáveis como tentativa de esclarecimento do significado da enurese para as crianças, tema abordado por um estudo com mais de 8.000 crianças inglesas de nove anos de idade (Butler \& Heron, 2007), que revelou que elas vêem a enurese como a oitava pior dificuldade da infância, ficando atrás apenas de eventos sociais mais problemáticos, como ser provocado pelos colegas, deixado de fora das brincadeiras e não ter amigos. A forma como os pais lidam com o problema pode variar e ser determinante para o impacto sofrido pela criança, uma vez que pode afetar o vínculo necessário e saudável entre pais e filhos. Neste sentido, é de grande importância lembrar os resultados do estudo de Schober, Lipman, Haltigan e Kuhn (2003) que ao compararem os portadores de enurese com crianças e 
adolescentes sem o transtorno, identificaram que a enurese pode levar a patologias relacionadas ao apego.

Butler (1994) propõe dois modelos de reação parental à enurese. O primeiro é caracterizado por uma sensação de desamparo que leva os pais, ainda que preocupados com os efeitos da enurese na criança, a atribuírem este distúrbio uma causa interna errônea, o sono pesado. Esses pais geralmente acordam a criança durante a noite e restringem os líquidos, contingências que apenas ajudam a manter a enurese. Essas são as duas estratégias mais utilizadas pelos pais de crianças portadoras de enureses (Butler, Golding, Heron \& ALSPAC Study Team, 2007). O segundo modelo é marcado pela intolerância. Nesses casos, a preocupação dos pais é menor com a criança e maior com eles próprios, especialmente em relação ao trabalho e encargos extras que a enurese acarreta. Há uma falsa crença de que o problema é controlável e a criança é culpada por ser preguiçosa, podendo ser punida fisicamente por seus pais.

Um estudo com 105 pais de crianças com enurese severa em Hong Kong (Ng \& Wong, 2004) mostrou que eles se sentem envergonhados, preocupados e com raiva, sendo que $57 \%$ admitiram punir seus filhos. Além disso, ter um filho que apresente o transtorno pode estar associado a um maior escore em escalas de depressão em comparação com mães de crianças que não o têm (Egemen, Akil, Canda, Ozyurt \& Eser, 2007). Pode-se ainda lembrar que pais intolerantes também tendem a desistir mais facilmente do tratamento (Morgan \& Young, 1975).

Fruto do reconhecimento da existência do impacto que a enurese causa sobre as crianças e adolescentes, Butler e McKenna (2002) realizaram um estudo com profissionais a fim de identificar quais as melhores formas de lidar com a intolerância e aumentar as chances de que o tratamento com o alarme, tido como o mais efetivo, seja bem sucedido. Os autores identificaram que a atitude mais indicada é fazer com que os pais entendam a enurese, ressaltando especialmente a ausência de culpa por parte da criança.

Sendo assim, é de vital importância para o trabalho clínico com crianças e adolescentes portadores de enurese a compreensão das especificidades do impacto sofrido e a avaliação da reação dos pais, especialmente no nível de tolerância ao problema. Considerando as diferenças culturais e a escassez de trabalhos sobre o assunto com populações brasileiras, o objetivo do presente trabalho, identificar e buscar relações entre os níveis de impacto e intolerância pode ser útil para diversos profissionais da área de saúde que lidam diariamente com esse tipo de quadro, a fim de que possam traçar estratégias efetivas para amenizar as consequências negativas da enurese para as crianças e suas famílias.

\section{MÉTODO}

\section{Amostra}

O estudo incluiu 126 crianças e adolescentes entre 5 e 17 anos, todos inscritos em um projeto que oferece atendimento específico para crianças e adolescentes com enurese (Projeto Enurese), independente de terem sido atendidos, e que preencheram os seguintes requisitos: (a) apresentar enurese; (b) ter a escala de impacto respondida pela criança ou adolescente e (c) ter a escala de tolerância respondida por pelo menos um dos pais.

A amostra foi composta por 41 meninas, com idade média igual a 9,68 (DP=2,70) e 85 meninos, com idade média igual a 9,45 (DP=2,67).

\section{Instrumentos}

Foram utilizados os seguintes instrumentos:

(a) Escala de Impacto, desenvolvida por Butler (1994). Essa escala, preenchida pela criança ou pelo adolescente, consiste de 17 afirmações, como "Minha mãe tem roupa demais para lavar", "Sinto-me diferente dos meus amigos" e "Meu pai ou minha mãe fica bravo(a) comigo". A estas sentenças são apresentadas respostas "não", "às vezes" e "sim", por meio das quais são calculados valores de graus de impacto. À resposta "não" pontua-se 0 pontos, à resposta "às vezes" pontua-se 1 ponto, e à resposta "sim" pontua-se 2 pontos. Quanto maior o escore total, maior é o impacto sofrido pela criança ou adolescente. A escala possui 8 fatores distintos, subdivididos em preocupações não psicológicas (consequências físicas, higiene, contingências, tempo na cama - itens 1 a 9) e preocupações psicológicas (falta de socialização, medo da descoberta, senso de diferença, reação emocional - itens 10 a 17). $O$ instrumento teve sua versão brasileira validada preliminarmente em um estudo que avaliou as propriedades psicométricas e unidimensionalidade da escala, com resultados satisfatórios (De Salvo, De Toni \& Silvares, 2008). Desse modo, além do impacto geral, também foram analisados os impactos psicológicos e não psicológicos causados pela enurese.

(b) Escala de Tolerância, desenvolvida por Morgan e Young (1975). Essa escala possui 20 itens para medir a tolerância/intolerância dos pais dos portadores de 
enurese. Os pais respondem "sim" ou "não" às afirmações, tais como "Eu já me acostumei com camas molhadas", "Eu tento ajudá-lo(a) a não se aborrecer com o xixi na cama", "Um bom tapa nunca fez mal a nenhuma criança que molhe a cama e pode fazer muito bem". O valor final é a mediana de todos os itens respondidos positivamente ("sim"), sendo que um alto escore indica intolerância por parte do pai ou da mãe em relação ao filho enurético. A versão original do instrumento aponta que os pais são considerados intolerantes quando o escore é maior do que 1,45.

\section{Procedimento}

Todas as crianças e adolescentes portadores de enurese primária inscritos no Projeto Enurese e que possuíam em seus prontuários a Escala de Impacto (Butler, 1994) e a Escala de Tolerância (Morgan \& Young, 1975) respondidos com a ajuda de uma estagiária de psicologia na entrevista inicial, foram analisados, permitindo calcular o grau de impacto geral, impacto psicológico e impacto não psicológico, além dos sentimentos de tolerância/ intolerância dos pais.

\section{Cuidados éticos}

O presente projeto de pesquisa faz parte de um de um projeto mais amplo, denominado "Efeito de Variáveis Moderadoras no Tratamento da Enurese", que foi submetido e aprovado pelo Comitê de Ética em Pesquisa do Instituto de Psicologia da Universidade de São Paulo (Of. 6306/CEPH-20/12/06). As famílias participantes assinaram um Termo de Consentimento Livre e Esclarecido, relativo ao projeto de atendimento clínico acima mencionado, no qual autorizaram o uso dos dados gerados durante a pesquisa para estudos científicos.

\section{RESULTADOS}

A Tabela 1 apresenta o número de respondentes, as médias e os desvios-padrão da Escala de Tolerância dos pais, o impacto total, psicológico e não psicológico medidos na Escala de Impacto, respondida pelos portadores de enurese. Cabe ressaltar que quanto maior for o grau da Escala de Tolerância, maior é a intolerância dos pais. As médias dos escores das escalas de tolerância preenchidas por pais e mães foram comparadas a fim de identificar diferenças estatisticamente significativas, assim como os escores de impacto psicológico e não psicológico.

TABELA 1

Valores médios dos escores das escalas utilizadas

\begin{tabular}{lcccc}
\hline \multicolumn{1}{c}{ Variável } & $\mathbf{N}$ & Média & Desvio Padrão & ${\text { Valor } \mathbf{p}^{*}}^{*}$ \\
\hline Tolerância mãe & 111 & 2,29 & 0,67 & $<.001$ \\
Tolerância pai & 46 & 2,47 & 0,68 & $<.001$ \\
\hline Impacto psicológico & 125 & 9,40 & 3,74 & 3,51 \\
Impacto não psicológico & 125 & 10,83 & 6,26 & \\
\hline Impacto total & 125 & 20,21 &
\end{tabular}

Nota. *Teste t.

Como é possível observar na Tabela 1, um maior número de mães respondeu a Escala de Tolerância que pais. Este dado pode ser explicado pelo fato delas acompanharem os filhos nas entrevistas de triagem, em que os instrumentos são aplicados, com maior frequência. Se comparadas às da amostra normativa americana, em que o instrumento foi desenvolvido, as médias da tolerância apresentadas acima são elevadas. A média das Escalas de Tolerância preenchidas pelos pais se mostrou significativamente mais elevada que a média das preenchidas pelas mães, apontando um maior nível de intolerância, uma vez que na escala, quando maior o escore, maior o nível de intolerância. Todas as crianças responderam a Escala de Impacto, e se pode observar que o impacto não psicológico se mostrou significativamente superior ao psicológico.

Foi verificado se havia correlação entre os escores obtidos nas escalas e idade, cujos resultados podem ser vistos na Tabela 2. 
TABELA 2

Correlação entre todas as escalas analisadas

\begin{tabular}{lccrrrr}
\hline & Idade & $\begin{array}{c}\text { Impacto } \\
\text { total }\end{array}$ & $\begin{array}{c}\text { Impacto não } \\
\text { psicológico }\end{array}$ & $\begin{array}{c}\text { Impacto } \\
\text { psicológico }\end{array}$ & $\begin{array}{c}\text { Tolerância } \\
\text { mãe }\end{array}$ & $\begin{array}{c}\text { Tolerância } \\
\text { pai }\end{array}$ \\
\hline Idade & 1 & $0,207^{*}$ & 0,144 & $0,213^{*}$ & $0,188^{*}$ & 0,196 \\
\hline Impacto total &, $207^{*}$ & 1 &, $848^{* *}$ & $0,873^{* *}$ &, $240^{*}$ &, 182 \\
\hline Impacto não psicológico &, 144 &, $848^{* *}$ & 1 &, $481^{* *}$ &, $209^{*}$ &, 259 \\
\hline Impacto psicológico &, $213^{*}$ &, $873^{* *}$ &, $481^{* *}$ & 1 &, $212^{*}$ &, 063 \\
\hline Tolerância mãe &, $188^{*}$ &, $240^{*}$ &, $209^{*}$ &, $212^{*}$ & 1 &, 241 \\
\hline Tolerância pai &, 196 &, 182 &, 259 &, 063 &, 241 & 1 \\
\hline
\end{tabular}

Nota. Correlação de Pearson. Legenda: * $p<0,05 ;{ }^{* *} p<0,01$.

Observaram-se correlações significativas entre diversos escores das escalas, com exceção da escala de tolerância preenchida pelos pais, que não apresentou correlação significativa com nenhum dos outros escores. Obviamente o impacto total se altera de acordo com o impacto psicológico e não psicológico, pois são escores do mesmo instrumento, sendo que um engloba os outros dois. Pode-se observar que quanto mais into- lerante é a mãe, maiores são os três níveis de impacto apresentados pela criança. Verificou-se também que tanto o impacto total como a intolerância da mãe são maiores de acordo com a idade mais elevada da criança. Os participantes foram divididos em grupos de acordo com o sexo e as médias dos escores foram comparadas a fim de verificar se houve diferenças entre eles, conforme mostra a Tabela 3.

TABELA 3

Comparação entre grupos divididos de acordo com o sexo

\begin{tabular}{|c|c|c|c|c|}
\hline & Sexo & Média & DP & Sig.* \\
\hline \multirow{2}{*}{ Impacto total } & Feminino & 20,37 & 6,23 & \multirow{2}{*}{0,94} \\
\hline & Masculino & 20,14 & 6,32 & \\
\hline \multirow{2}{*}{ Tolerância mãe } & Feminino & 2,32 & 0,67 & \multirow{2}{*}{0,60} \\
\hline & Masculino & 2,29 & 0,69 & \\
\hline \multirow{2}{*}{ Tolerância pai } & Feminino & 2,62 & 0,71 & \multirow{2}{*}{0,56} \\
\hline & Masculino & 2,39 & 0,66 & \\
\hline \multirow{2}{*}{ Impacto não psicológico } & Feminino & 10,88 & 3,39 & \multirow{2}{*}{0,62} \\
\hline & Masculino & 10,81 & 3,60 & \\
\hline \multirow[t]{2}{*}{ Impacto psicológico } & Feminino & 9,49 & 4,08 & \multirow{2}{*}{0,86} \\
\hline & Masculino & 9,36 & 3,59 & \\
\hline
\end{tabular}

Nota. ${ }^{*}$ Teste T para amostras independentes.

Como pode ser visto na Tabela 3, não houve diferenças entre os escores médios alcançados pelos grupos de meninos e meninas. Isso indica que as crianças de ambos os sexos são impactadas igualmente e os pais de meninos e meninas são intolerantes da mesma forma.

\section{DISCUSSÃO}

Foi possível perceber que os pais se mostraram significativamente mais intolerantes que as mães, apesar do contrário ser mais esperado, uma vez que se supõe que as mães tenham mais contato com o pro- blema e trabalho extra, o que pode inclusive levá-las a apresentar maiores índices de depressão (Egemen \& cols., 2008). Esses dados podem sugerir então que a tendência dos pais estudados pode ser a do padrão descrito por Butler (1994) em que eles estão mais preocupados consigo mesmos e com os encargos que a enurese traz para eles, do que com os próprios filhos. O autor aponta ainda, que esses pais acreditam que o problema apenas acontece porque os filhos são preguiçosos e por isso não controlam a urina, um relato comum na experiência clínica dos autores. 
Foi possível perceber, a partir do impacto não psicológico, que as questões que mais incomodam as crianças são, por exemplo, o fato de não poderem permanecer mais tempo na cama por ela estar molhada ou ter que tomar banho sempre que levantam, e não as consequências sociais, emocionais e de autoimagem citadas na literatura (Butler, 2001).

A relação entre o impacto e a tolerância corrobora ao observado no estudo de Schober e cols. (2004), que mostra que a tolerância dos pais com relação ao molhar a cama pode trazer maior ou menor impacto para a criança. Se os pais se mostram muito intolerantes, os filhos podem manifestar patologias relacionadas ao apego, afetando o vínculo estabelecido, o que pode levar a problemas de comportamento e outras dificuldades emocionais no futuro. A relação entre o maior grau de impacto e intolerância de acordo com a idade do enurético pode ser confirmada no estudo de Theunis e cols. (2002). Embora não seja possível estabelecer relações de causalidade entre esses dados, pode-se hipotetizar que a intolerância aumenta pelo contato continuado da mãe com as dificuldades trazidas pelo problema. Pode-se, com isso, supor que o impacto, para a criança com enurese, se torna maior, uma vez que ela precisa lidar com a reação negativa dos pais e com as restrições na vida social, que tendem a ser mais significativas com o avanço da idade.

Ainda nesta linha, pode-se hipotetizar que o maior grau de impacto e intolerância nas crianças mais velhas, se deve pelo fato de que o transtorno, ao se estender por muitos anos, pode acentuar os problemas de comportamento apresentados. Sendo assim, fortalece-se a tese de Santos e Silvares (2006) de que a enurese deve ser encarada como um problema primário e que a sua resolução tem uma função preventiva em relação a outras dificuldades que a família pode enfrentar. Foi possível perceber que possivelmente a atitude da mãe cause mais impacto nos filhos que a do pai, tendo em vista que mesmo que os pais sejam mais intolerantes, o impacto sentido pelos filhos é mais afetado pela intolerância da mãe. Essa maior suscetibilidade dos filhos com relação às atitudes da mãe pode ser apoiada também no fato de que as mães das crianças com o transtorno têm maiores pontos nas escalas de depressão que as mães das crianças sem enurese (Egemen \& cols., 2008).

De acordo com os estudos de Collier e cols. (2002), os meninos se mostraram mais impactados que as meninas, mas no presente estudo essa diferença não foi encontrada. No entanto, os instrumentos utiliza- dos pelos referidos autores não foram idênticos aos utilizados na presente pesquisa, uma vez que as crianças no estudo citado não responderam a um questionário sobre o impacto da enurese em si, e sim a um inventário sobre o autoconceito.

A partir dos dados apresentados e discutidos, pode-se considerar que o objetivo principal desta pesquisa foi atingido, verificando-se a relação entre a intolerância dos pais e o impacto relatado pelos filhos. Ressalta-se que, mesmo que a intolerância paterna seja superior, a intolerância materna foi a única que teve uma correlação positiva significante com o impacto sentido pelos filhos, o que talvez se explique pelo número menor de questionários respondidos pelos pais.

Os objetivos específicos também foram alcançados, levando em consideração que: (a) o impacto aumenta de acordo com a idade, sendo ligado à intolerância da mãe e muito parecido entre os grupos feminino e masculino, embora se mostre um pouco maior no grupo feminino; (b) a intolerância dos pais é maior que a das mães e ambos são mais intolerantes com as meninas, embora essa diferença seja pequena entre os sexos; (c) a intolerância materna parece impactar mais os filhos que a paterna, sendo que a segunda tem maior grau de interferência no impacto não psicológico, enquanto a primeira interfere mais no impacto psicológico; (d) o impacto psicológico e a intolerância materna aumentam de acordo com a idade, o impacto sentido por ambos os sexos é muito parecido, e a intolerância dos pais para com os dois grupos também se assemelha muito.

Os resultados obtidos mostram a necessidade de se entender a enurese como um problema familiar, avaliando não apenas o comportamento alvo, mas também as implicações para a criança e sua família. Uma vez que os tratamentos costumam ser eficazes, a compreensão da dinâmica familiar acerca do problema é um dos aspectos fundamentais do trabalho do profissional de saúde com esse tipo de quadro. A elevação dos índices de intolerância e impacto de acordo com a idade evidencia, ainda, a necessidade de se tratar a enurese cedo a fim de evitar outros problemas. Um estudo importante a se fazer futuramente seria aplicar novamente as escalas de Tolerância e de Impacto nos pais e nas crianças que foram submetidas ao tratamento da enurese para averiguar quais mudanças podem aparecer em decorrência do tratamento.

As limitações da pesquisa se encontram no fato de que o número de mães que participaram da pesquisa ser bastante superior ao número de pais, pois são elas 
que geralmente acompanham os filhos até a entrevista de triagem. Seria importante desenvolver uma estratégia que garantisse o retorno dos questionários também por parte dos pais. Outro ponto limitador que deve ser levado em conta é que todos os dados são baseados em questionários e seus resultados podem sofrer pressão da situação em que os clientes se encontram. Outro possível obstáculo é o fato de que muitas vezes as pessoas não entendem corretamente as questões levantadas pelas escalas, levando-as a responder de forma errada. $\mathrm{O}$ constante aprimoramento dos instrumentos e das formas de aplicação pode amenizar esses dois últimos pontos.

\section{REFERÊNCIAS}

Achenbach, T., \& Rescorla, L. (2001). Manual for the ASEBA school-age forms \& profiles. Burlington, VT: ASEBA.

American Psychiatric Association. (2002). Manual diagnóstico e estatístico de transtornos mentais ( $4^{\mathrm{a}}$ ed.). Porto Alegre: Artmed.

Baeyens, D., Roeyers, H., Naert, S., Hoebeke, P., \& Walle, J. (2007). The impact of maturation of brainstem inhibition on enuresis: A startle eye blink modification study with 2-year followup. The Journal of Urology, 178, 2621-2625.

Butler, R. J. (1994). Nocturnal enuresis: The child's experience. Oxford: Butterworth-Heinemann.

Butler, R. J. (1998). Night wetting in children: Psychological aspects. Journal of Child Psychiatry, 39, 453-463.

Butler, R. J. (2001). Impact of nocturnal enuresis on children and young people. Scandinavian Journal of Urology and Nephrology, 35, 169-176.

Butler, R. J. (2004). Childhood nocturnal enuresis: Developing a conceptual framework. Clinical Psychology Review, 24, 909931.

Butler, R. J., \& Heron, J. (2007). An exploration of children's views of bed-wetting at 9 years. Child: Care, Health and Development, 34, 65-70.

Butler, R. J., \& Holland, P. (2000). The three systems: A conceptual way of understanding nocturnal enuresis. Scandinavian Journal of Urology and Nephrology, 34, 270-277.

Butler, R. J., \& McKenna, S. (2002). Overcoming parental intolerance in childhood nocturnal enuresis: A survey of professional opinion. British Journal of Urology International, 89, 295-297.

Butler, R. J., Golding, J., Heron, J., \& ALSPAC Study Team. (2007). Nocturnal enuresis: A survey of parental coping strategies at 71/2 years. Child: Care, Health \& Development, 31, 659667.

Butler, R. J., Golding, J., Northstone, K., \& ALSPAC Study Team. (2005). Nocturnal enuresis at 7.5 years old: Prevalence and analysis of clinical signs. British Journal of Urology International, 96, 404-410.

Collier, J., Butler R. J., Redsell, S., \& Evans, J. (2002). An investigation of the impact of nocturnal enuresis on children's selfconcept. Scandinavian Journal of Urology and Nephrology, 36, 204-208.
De Salvo, C., De Toni. P., \& Silvares, E. (2008). Análise fatorial e unidimensionalidade da escala de impacto à enurese. Psico, 39, 240-245.

Egemen, A., Akil, I., Canda, E., Ozyurt, B., \& Eser, E. (2008). An evaluation of quality of life of mothers of children with enuresis nocturna. Pediatric Nephrology, 23, 93-98.

Houts, A. C. (1991). Nocturnal enuresis as a biobehavioral problem. Behavior Therapy, 22, 33-51.

Houts, A. C. (2003). Behavioral treatment for enuresis. Em A. E. Kazdin \& J. R. Weisz (Orgs.), Evidence-based psychotherapies for children and adolescents (pp. 389-406). New York: Guilford Press.

Jensen, N., \& Kristensen, G. (2001). Frequency of nightly wetting and the efficiency of alarm treatment of nocturnal enuresis. Scandinavian Journal of Urology and Nephrology, 35, 357363.

Joinson, C., Heron, J., \& Butler, R. J. (2007). Psychological problems in children with bedwetting and combined (day and night) wetting: A UK population-based study. Journal of Pediatric Psychology, 32, 605-616.

Longstaffe, S., Moffatt, M. E., \& Whalen, J. C. (2000). Behavioral and self-concept changes after six months of enuresis treatment: A randomized, controlled trial. Pediatrics, 105, 935-940.

Morgan, R. T., \& Young, G. C. (1975). Parental attitudes and the conditioning treatment of childhood enuresis. Behavior Research and Therapy, 13, 197-199.

Mota, D. M., Victora, C. G., \& Hallal, P. C. (2005). Investigação de disfunção miccional em uma amostra populacional de crianças de 3 a 9 anos. Jornal de Pediatria, 81(3), 225-32.

Nevéus, T. (2009). Diagnosis and management of nocturnal enuresis. Current Opinion in Pediatrics, 21(2), 199-202.

Nevéus, T., Gontard, A., Hoebeke, P., Hjälmås, K., Bauer, S., \& Bower, W. e cols. (2006). The standardization of terminology of lower urinary tract function in children and adolescents: Report from the Standardisation Committee of the International Children's Continence Society. The Journal of Urology, 176, 314-324.

Ng, C. F., \& Wong, S. N. (2004). Primary nocturnal enuresis: Patient attitudes and parental perceptions. Hong Kong Journal of Paediatrics, 9, 54-58.

Pereira, R. F., Del Prette, Z. A., \& Silvares, E. (2008). Problemas de comportamento em enuréticos: Análise do índice de mudança confiável (IMC). Arquivos Brasileiros de Psicologia, 60(3), 129-141.

Robinson, J. Butler, R. J., Holland, P., \& Doherty-Williams, D. (2003). Self-construing in children with primary monosymptomatic nocturnal enuresis. Scandinavian Journal of Urology and Nephrology, 37, 124-128.

Rocha, M., Costa, N., \& Silvares, E. (2008). Changes in parents' and self-reports of behavioral problems in Brazilian adolescents after behavioral treatment with urine alarm for nocturnal enuresis. International Brazilian Journal of Urology, 34, 74957.

Santos, E., \& Silvares, E. (2006). Crianças enuréticas e crianças encaminhadas para clínicas-escola: Um estudo comparativo da percepção de seus pais. Psicologia: Reflexão e Crítica, 19, 277-282.

Schober, J., Lipman, R., Haltigan, J., \& Kuhn, P. (2004). The impact of monosymptomatic nocturnal enuresis on attachment 
parameters. Scandinavian Journal of Urology and Nephrology, $38,47-52$.

Schoen-Ferreira, T., Marteleto, M., Medeiros, E., Fisberg, M., \& Aznar-Farias, M. (2007). Levantamento de enurese no município de São Paulo. Revista Brasileira de Crescimento e Desenvolvimento Humano, 17(2), 31-36.

Theunis, M., Van Hoecke, E., Paesbrugge, S., Hoebeke, P., \& Walle, J. (2002). Self-image and performance in children with nocturnal enuresis. European Urology, 41, 660-667.

Van Hoeck, K., Bael, A., Lax, H., Hirche, H., Bernaerts, K., Vandermaelen, V., e cols. (2007). Improving the cure rate of alarm treatment for monosymptomatic nocturnal enuresis by increa- sing bladder capacity: A randomized controlled trial in children. The Journal of Urology, 179, 1122-1127.

Van Hoecke, E., Hoebeke, P., Braet, C., \& Walle, J. (2004). An assessment of internalizing problems in children with enuresis. The Journal of Urology, 171, 2580-2583.

\section{Sobre os autores:}

Recebido: $31 / 01 / 2010$

Última revisão: $14 / 07 / 2010$

Aceite final: $20 / 08 / 2010$

Yasmin Spaolonzi Daibs: Graduanda em psicologia pela Universidade Presbiteriana Mackenzie. Aprimoranda do Instituto de Psicologia da Universidade de São Paulo.

Rodrigo Fernando Pereira: Psicólogo, mestre em Psicologia Clínica pela Universidade de São Paulo (2006) e doutor em Psicologia Clínica pela Universidade de São Paulo (2010).

Deisy Ribas Emerich: Graduada em psicologia pela Universidade Presbiteriana Mackenzie e mestranda em Psicologia Clínica pela Universidade de São Paulo.

Edwiges Ferreira de Mattos Silvares: Psicóloga, mestre em Psicologia Experimental pela Northeastern University, doutora em Psicologia (Psicologia Experimental) pela Universidade de São Paulo, professora titular do Departamento de Psicologia Clínica do Instituto de Psicologia da Universidade de São Paulo.

Endereço para correspondência: Edwiges Ferreira de Mattos Silvares - Av. Prof. Mello Moraes, 1.721 - Bloco F - Sala 30 - Cidade Universitária - 05508-030 São Paulo/SP. Endereço eletrônico: efdmsilv@usp.br. 